\title{
A PROPÓSITO DE LOS DESASTRES NATURALES, EL CAMBIO CLIMÁTICO Y LA PROTECCIÓN DE LOS DERECHOS HUMANOS. REFLEXIONES, REALIDADES Y DESAFÍOS
}

Juan Pablo Terminiello

\section{Resumen}

Este artículo pretende introducir al lector en la relación que se plantea entre los desastres naturales y el cambio climático con los esfuerzos para asegurar el goce y ejercicio de los derechos humanos de las personas afectadas. Acudiendo a las experiencias y lecciones aprendidas de los grandes desastres de la última década y a algunas iniciativas desarrolladas en el marco de la acción humanitaria, se pretende presentar un panorama de cómo éstos fenómenos impactan en la protección de los derechos humanos, señalando aquellos derechos y situaciones de vulnerabilidad de particular relevancia. Asimismo, se busca introducir uno de los principales desafíos humanitarios que plantean los desastres y el cambio climático, la situación legal y de protección de las personas forzadamente desplazada como consecuencia de sus efectos adversos.

\section{Summary}

The present article aims to introduce the leader into a relationship between natural disasters and el imate change with the efforts to ensure the enjoyment and exercise of human rights of those affected. Turning to the experiences and lessons learnt from the great disasters in the last decade and some initiatives developed within the framework of humanitarian action, it is expected to present an outlook of how these phenomenons qffect the protection of human rights. pointing out those rights and situations of vulnerability that have a particular relevance. It also seeks to introduce one of the most principie humanitarian challenges presented bv the natura! disasters and the climate change, legal status and protection of people displaced due to its adverse effeets.

\section{Sommaire}

Le présent article vise ¿i présenter au lecteur la relation qui nait entre les catastrophes naturelles et le changement climatique avec des efforts pour assurer la jouissance et l'exercice des droits humains. $S$ 'inspirant des experiences et des ie<;ons tirées des catastrophes majeures de la derniére décennie et quelques initiatives dans le cadre de $i$ action humanitaire est de fournir un aperqu de la maniere dont ces phénoménes d'impact sur la protection des droits de $i$ 'homme, soulignant les droits et les situations vulnérabilité de pertinence particuliére. II vise également á introduire un des défis majeurs poses par les catastrophes humanitaires et le changement climatique, le statut juridique et la protection des personnes déplacées de forcé en raison de ses effets néfastes. 


\section{INTRODUCCIÓN}

Las prácticas autoritarias, los conflictos armados, tanto internos como internacionales, la violencia, la discriminación y la pobreza continúan siendo algunas de las principales amenazas al goce y ejercicio de los derechos humanos de vastos sectores de la humanidad. Sin embargo, asoman nuevas amenazas que asociadas con algunas de la situaciones ya existentes se presentan como un serio desafío a la tarea de proteger los derechos humanos.

El cambio climático y los desastres naturales se inscriben entre estas nuevas amenazas que asociadas a la violencia, los conflictos y la pobreza afectan el goce de los derechos humanos de cientos de miles de personas en todo el mundo. La protección de las personas afectadas por los desastres naturales y los efectos adversos del cambio climático constituye un delicado desafío humanitario que planeta una clara dimensión de derechos humanos.

Los desastres naturales han sido tradicionalmente concebidos como un desafío logístico principalmente abordado a través de las operaciones de rescate y la distribución de asistencia de emergencia tal como agua potable, abrigo y medicamentos. Poca o nula atención se ha dado desde la Academia y desde la práctica de las agencias humanitarias al impacto devastador que estos fenómenos pueden tener en el goce y ejercicio de los derechos humanos fundamentales de las personas afectadas.

La experiencia de los grandes desastres naturales ocurridos en años recientes, tales como el tsunami en el océano índico (2004), las inundaciones en Pakistán, el terremoto de Haití (2010) y la sequía en el cuerno de África (2011), han puesto en evidencia el efecto devastador que los desastres naturales pueden tener en el goce y ejercicio de los derechos humanos de las personas afectadas. Las personas desplazadas por los desastres naturales suelen enfrentar dificultades bastante similares a las que padecen las personas afectadas por los conflictos armados: pérdida y separación del grupo familiar; pérdida de la vivienda; pérdida y destrucción de documentación y propiedad; amenazas a su derecho a la vida, integridad y seguridad personal; exposición a la violencia sexual y de género, entre otras ${ }^{96}$.

En muchos casos, la amenaza a los derechos humanos de las personas afectadas no procede del evento natural en sí mismo sino de una inadecuada planificación y respuesta frente al desastre. A modo de ejemplo se puede señalar que la exposición a la violencia sexual y de género a la que se ven expuestas las mujeres y niñas en los campamentos y centros de albergue de emergencia, que constituye una de las más graves amenazas a sus derechos humanos, no responde de modo directo al terremoto $o$ inundación que pudo haber forzado su desplazamiento sino a la incapacidad/desinterés de las autoridades de gestionar y adoptar las medidas necesarias para la prevención y respuesta a este tipo de violencia. Las amenazas a los derechos humanos se potencian frente a la ausencia de un enfoque basado en derechos en la labor de las agencias responsables durante las fases de preparación y respuesta a la emergencia.

Para una apreciación más exacta del impacto de los desastres en los derechos humanos es preciso tener en cuenta que se estima que en 2010, más de 300 millones de personas en todo el mundo se vieron afectadas por los efectos de 373 desastres 
naturales registrados a lo largo del año. Esta cifra incluye un estimado total de las personas, hombres mujeres, niños y niñas, que en 2010 necesitaron de algún tipo de asistencia inmediata durante un periodo de emergencia tal como agua, alimento, albergue, saneamiento y/o asistencia médica. También incluye a las personas desplazadas como consecuencia de los desastres. Durante este mismo periodo se estima que más de 390,000 personas fallecieron como consecuencia de desastres naturales. ${ }^{97}$

Las personas afectadas por los desastres naturales como terremotos, tsunamis, inundaciones o sequías, suelen experimentar limitaciones y serios obstáculos al ejercicio de sus derechos humanos. Los desastres afectan la organización y estructura de la comunidad y hacen sentir fuertemente su impacto en áreas como la seguridad, el acceso a alimento, agua y saneamiento, la atención de la salud, el empleo, la educación y la vivienda. Producen muertes y daños que debieran ser mitigados desde la actuación de las autoridades como parte de la obligación genérica de los Estados de respetar y garantizar los derechos humanos de las personas sujetas a su jurisdicción. La ocurrencia de un desastre no libera ni condona al Estado y sus agentes de las obligaciones contraídas en el marco del derecho internacional de los Derechos Humanos, particularmente, si tenemos en cuenta que muchas de las acciones para la protección de tales derechos debieran ser adoptadas en la fase de preparación y mitigación de riesgos como instancias previas a la ocurrencia y respuesta frente a los desastres.

Como se analizará a lo largo de este trabajo, los desastres naturales exacerban los patrones de discriminación y exclusión que se encontraban presentes en las comunidades afectadas antes de la ocurrencia del evento y potencian las vulnerabilidades interfiriendo de modo decisivo en el goce y ejercicio de una amplia gama de derechos, tanto de los denominados derechos civiles y políticos como de los derechos económicos sociales y culturales. Aquello grupos de por sí vulnerables antes de la ocurrencia del desastre deberán enfrenar con posterioridad a su ocurrencia aún mayores obstáculos y limitaciones para el acceso y ejercicio de sus derechos.

La clara relación que existe entre los desastres naturales, y la afectación del goce y ejercicio de los derechos humanos no es igualmente fácil de identificar en el caso de los efectos adversos de proceso lento que conlleva el cambio climático. La dificultad de aislar en el tiempo el impacto de los efectos de proceso lento asociados al cambio climático, como la desertificación o la erosión de las costas marinas, no los priva, sin embargo, de su capacidad para interferir en el goce y ejercicio de los derechos de las personas y comunidades afectadas.

En 2008, el Consejo de Derechos Humanos de las Naciones Unidas adoptó una Resolución que reconoció categóricamente el impacto del cambio climático en la protección de los derechos humanos. La Resolución 7/23 afirma que "el cambio climático crea una amenaza inmediata y de gran alcance para la población y las comunidades de todo el mundo y tiene repercusiones sobre el pleno disfrute de los derechos humanos"98. Disasters in 2010. The Brookings Institution - London School of Eeonomics Project on Intemal Displacement, April 2011, disponible en: http://www.brookings.edu/--/media/Files/rc/repoi1s/2011/04 nd living dangerously/04nd living dangerously.pdf.

98

Naciones Unidas Consejo de Derechos Humanos, Resolución 7/23 Derechos humanos y cambio climático, 41 “ sesión, 28 de marzo de 2008. 
Al margen de los derechos que se podrán ver afectados por los efectos de los desastres naturales que como consecuencia del cambio climático habrán de aumentar en frecuencia e intensidad, existen derechos amenazados cuya vigencia se ve amenazada por los efectos del cambio climático de proceso lento. El derecho a la vida, el derecho a una alimentación adecuada, el derecho al agua, el derecho a la salud, el derecho a una vivienda adecuada y el derecho a la libre determinación se encuentran entre los derechos humanos que se verían severamente amenazados directa 0 indirectamente por las consecuencias del cambio climático de acuerdo al Informe que en enero de 2009 la Oficina del Alto Comisionado de las Naciones Unidas para los Derechos Humanos presentó ante el Consejo de Derechos Humanos".

Los desastres naturales y los efectos adversos del cambio climático se plantean como una seria amenaza a la vigencia de los derechos humanos. Una de las más complejas consecuencias humanitarias de los desastres y el cambio climático se da en el campo del desplazamiento forzado de personas. Los desastres naturales y el cambio climático se han convertido en factores determinantes del desplazamiento forzado de personas junto a la persecución por motivos étnicos, políticos o religiosos y los conflictos armados. Al respecto, el Alto Comisionado de las Naciones Unidas para los Refugiados (ACNUR) ha dicho que "la influencia del cambio medioambiental en la movilidad humana es ostensible y creciente" ${ }^{100}$.

Este artículo pretende introducir al lector en la relación que se plantea entre los desastres naturales y el cambio climático con los esfuerzos para asegurar el goce y ejercicio de los derechos humanos de las personas afectadas. Acudiendo a las experiencias y lecciones aprendidas de los grandes desastres de la última década y a algunas iniciativas desarrolladas en el marco de la acción humanitaria, se pretende presentar un panorama de cómo éstos fenómenos impactan en la protección de los derechos humanos, señalando aquellos derechos y situaciones de vulnerabilidad de particular relevancia. Asimismo, se busca introducir uno de los principales desafíos humanitarios que plantean los desastres y el cambio climático, la situación legal y de protección de las personas forzadamente desplazada como consecuencia de sus efectos adversos.

\section{LA PROTECCIÓN DE LOS DERECHOS HUMANOS EN CONTEXTO DE DESASTRES NATURALES}

Al margen de las discusiones que se puedan librar acerca de su naturaleza y fundamento, los derechos humanos han sido cristalizados en una serie de instrumentos internacionales, tanto universales como regionales, que implican "no solamente la consagración legal de los derechos subjetivos necesarios para el normal desarrollo de la vida del ser humano en sociedad que el Estado debe respetar y garantizar, sino el reconocimiento de que la responsabilidad del Estado queda comprometida en caso de violación no reparada" ${ }^{101}$. los derechos humanos, A/HRC/10/61, 15 de enero de $2(109$.

100 Alto Comisionado de las Naciones Unidas para los Refugiados (ACNUR), InSearch ofShelter: Mapping the Effects of Climate Change on Human Migration and Displacement, Mayo de 2009, disponible en: http://www.unhcr.org/refworld/docid/4ddb65eb2.html.

101 Pinto. Ménica, Temas de Derechos Humanos, Editores del Puerto. Buenos Aires, Argentina, 2004. pág 10. 
Entre los derechos reconocidos bajo los catálogos internacionales de derechos humanos actualmente vigentes y vinculantes" ${ }^{12}$ se encuentran derechos civiles y políticos como el derecho a la vida, la libertad y seguridad personal, la prohibición de la tortura, otros tratos o penas crueles, inhumanos o degradantes, la libertad de circulación, la libertad de expresión, de asociación y la libertad religiosa, entre otros. Los pactos internacionales de derechos humanos también han incorporado los denominados derechos económicos, sociales y culturales, como el derecho a la educación, al trabajo, a la salud, a la vivienda, a una alimentación adecuada, y recientemente también se ha reconocido el derecho al agua potable y el saneamiento como un derecho humano esencial para el pleno disfrute de la vida y de todos los derechos humanos 103.

La protección de los derechos humanos implica, siguiendo la siguiendo la definición adoptada por el Comité Permanente entre Organismos (1ASC. por sus siglas en inglés $)^{104}$, "todas las actividades tendientes a conseguir el pleno respeto de los derechos de las personas de conformidad con la letra y el espíritu de la normativa pertinente (derechos humanos, derecho humanitario y derecho de los refugiados)" 105.

En el contexto de los desastres naturales, las actividades de protección de derechos humanos pueden abarcar un abanico amplio de acciones e iniciativas que pueden ir desde la instalación de letrinas y la distribución de agua potable, vestido y alimento, hasta el desarrollo de actividades de abogacía y monitoreo, como así también el trabajo con las propias personas afectadas para el desarrollo de acciones de prevención y respuesta a la violencia sexual y de género en los centros de albergue y campamentos. En la respuesta al desastre, la noción de protección se desprende de toda formalidad para tomarse flexible y abarcar un pluralidad de medidas esencialmente pragmáticas que tienen como objetivo prevenir la violación o restablecer el ejercicio de derechos fundamentales que pudieron verse afectados a consecuencia del desastre. A modo de ejemplo se puede señalar como la simple acción de mejorar la iluminación en ciertas áreas de los campamentos o la separación de los baños de hombres y mujeres devienen acciones claramente protectorías a fin de asegurar el derecho a la seguridad personal y a una vida libre de violencia de género de las mujeres y niñas.

Las personas afectadas por los desastres naturales permanecen por lo general dentro de su país de origen y, en consecuencia, tienen derecho a gozar de la protección de todos los derechos y garantías que le reconocen las normas internacionales de derechos vinculante y exigible para los Estados Parte. Igualmente, la jurisprudencia y la doctrina han reconocido en numerosas ocasiones el carácter de ius cogens de algunas de sus disposiciones, lo que las toma vinculantes frente a todos los Estados independientemente de que hubieran ratificado o no las convenciones. A nivel regional se deben destacar la Convención Americana sobre Derechos Humanos (CADH.1\%9) y su protocolo Adicional en materia de Derechos Económicos, Sociales y Culturales ("Protocolo de San Salvador").

103 A/64/L.63/Rev.I, 26 de julio de 2010.

Cfr. Naciones Unidas. Asamblea General, El derecho humano al agua y el saneamiento,

104EI 1ASC fue establecido por la Resolución 46/182 de la Asamblea General de las Naciones Unidas como una medida para el fortalecimiento de la coordinación de la asistencia humanitaria de emergencia. Funciona como un foro inter agencia para la coordinación, desarrollo de políticas y toma de decisiones que involucra a las principales agencias de las Naciones Unidas y otras agencias humanitarias.

105

Cfr. IASC, Directrices Operacionales sobre la protección de las personas en situaciones de desastres naturales, Proyecto de Brookings - Bem sobre Desplazamiento Interno, Mayo de 2011, pág.

5, disponible en: http://wwvv.brookings.edU/ /media/Files/rc/reports/201 1/0106_operational_guidelines_ nd/0106_operational_guidelines_nd_spanish.pdf. 
humanos y de derecho internacional humanitario que puedan resultar vinculantes para el Estado de que se trate.

El Estado continúa siendo el principal responsable de proteger los derechos humanos de las personas sometidas a su jurisdicción, incluidas, por supuesto, las personas que se puedan ver afectadas y desplazadas internamente como consecuencia de los efectos adversos de los desastres naturales. Corresponde al Estado no sólo la obligación de respetar los derechos humanos de todas las personas que viven en su territorio o jurisdicción, incluidos los desplazados, sino también de proteger y garantizar el goce y ejercicio de esos derechos. En relación a los desastres naturales, "las normas internacionales de derechos humanos obligan a los Estados a impedir o por lo menos mitigar los efectos negativos de los peligros naturales $y$, en particular, a proteger a las personas desplazadas por desastres naturales de los abusos y violaciones de los derechos humanos en las etapas de emergencia, recuperación y reconstrucción" 106

Aún en el marco de un desastre, "las personas no pierden los derechos de la población en general"107 y como contracara los Estados no pueden desentenderse de sus obligaciones para la protección de tales derechos.

\subsection{Desafíos v Enfoques}

Como se mencionó en la introducción a este trabajo, la falta de atención a la protección de derechos humanos de las personas en el contexto de los desastres naturales no es forzosamente deliberada, sino que con frecuencia responde a la aplicación de políticas inadecuadas, la incapacidad o sencillamente la desidia o descuido del Estado. Frente a esta realidad, "resultan fundamentales la reducción, la mitigación y la preparación respecto de los desastres, orientadas por un análisis contextual basado en los derechos humanos"108.

Aún en algunas de las principales iniciativas desarrolladas por la Comunidad Internacional en materia de mitigación de riesgos frente a desastres, el tema de la protección de los derechos humanos continúa recibiendo poca o nula atención. Es el caso por ejemplo del Marco de Acción de Hyogo 2005-2015 ${ }^{109}$ que a pesar de ser uno de los principales marcos estratégicos para incrementar la resiliencia de las comunidades y naciones frente a los desastres, y ser un instrumento adoptado con el auspicio de las

106 Consejo de Derechos Humanos de las Naciones Unidas, Informe del Representante del Secretario Genera! sobre los derechos humanos de Ios desplazados internos, Walter Kcilin: Adición: Protección de los desplazados internos en situaciones de desastres naturales, A/HRC/10/13/Add.I. 5 de marzo de 2009, párrafo 9.

107 IASC, Protección de las personas afectadas por los desastres naturales. Directrices Operacionales del Comité Permanente entre Organismos (IASC) sobre la protección de los derechos humanos en situaciones de desastres naturales. Proyecto de Brookings-Bem sobre Desplazamiento Interno, junio de 2006.

108 Consejo de Derechos Humanos de las Naciones Unidas, Informe del Representante del Secre tario General sobre los derechos humanos de los desplazados internos, op. cit., párrafo 5.

$109 \quad$ El Marco de Acción de Hyogo (MAH) es el instrumento más importante para la implementa-

ción de la reducción del riesgo de desastres que adoptaron los Estados miembros de las Naciones Unidas. E MAH está dirigido a aumentar la resiliencia de las naciones y las comunidades y lograr una reducción considerable de las pérdidas que ocasionan los desastres, tanto en términos de vidas humanas como en cuanto a los bienes sociales, económicos y ambientales. Lamentablemente, las pocas referencias incluidas en el MAH en términos de protección resultan de un carácter bastante general y omiten cualquier referencia o remisión a las normas internacionales de derechos humanos o la importancia de aplicar en las tareas de prevención y respuesta un enfoque basado en derechos. 
Naciones Unidas, contiene pocas referencias al tema de la protección de los derechos humanos entre los principios y acciones dirigidas a reducir los costos humanos y sociales de los desastres.

El descuido o desatención por el tema de la protección de los derechos humanos en situaciones de desastre natural también podría en ciertos casos verse favorecidas por la falta de capacitación en la materia de los actores que tradicionalmente se han vinculado a estas tareas, muchas veces asociados a las fuerzas armadas o de seguridad de los Estados afectados. Las acciones de prevención, mitigación y coordinación de la respuesta a los desastres naturales continúan en muchos casos en manos de Agencias del Estado sumamente aptas para la coordinación e implementación de acciones de asistencia humanitaria pero que no se encuentran familiarizadas con un enfoque y el lenguaje propio de los derechos humanos.

Si bien los militares resultan en muchos casos ser la agencia mejor equipada y preparada para la respuesta inicial y el desarrollo de la logística necesaria en operaciones de rescate y asistencia humanitaria frente a desastres naturales, W. Kalin señala que "el control militar de la ayuda y de los campamentos también puede poner en peligro a los beneficiarios ya que puede aumentar la vulnerabilidad de los desplazados internos a la explotación sexual y el abuso, así como (...) desalentar la capacidad de las personas desplazadas de controlar las decisiones que afectan su vida" ${ }^{110}$.

A partir de su advertencia sobre el impacto que en términos de protección de derechos puede conllevar militarizar la respuesta a los desastres naturales, Kalin nos introduce en una de las dimensiones más relevantes de la protección de los Derechos Humanos en el contexto de desastres naturales, la necesidad de aplicar un enfoque comunitario. Las personas afectadas por un desastre natural continúan siendo sujetos de derechos y no meros espectadores o beneficiarios del accionar humanitario. En este sentido, "al reconocer que todos los miembros de la comunidad son actores en el proceso de toma de decisiones, el enfoque comunitario refuerza la dignidad y autoestima de las personas y promueve su empoderamiento"111. Las personas afectadas deben ser incorporadas como actores en las tareas de prevención y respuesta. Su intervención es de sumo valor para la identificación de los riesgos de protección y el desarrollo de las estrategias de mitigación y respuesta.

Otro desafío fundamental en la tarea de proteger los derechos humanos de las personas afectadas por los desastres naturales radica en poder identificar que no todos los miembros de una comunidad sobrellevan de igual manera las limitaciones y amenazas que la situación de desastre impone al goce y ejercicio de los derechos humanos. Los desastres naturales exacerban los patrones de discriminación y exclusión que se encontraban presentes antes de la ocurrencia del evento y potencian las vulnerabilidades interfiriendo de modo decisivo en el goce y ejercicio de una amplia gama de derechos, tanto de los denominados derechos civiles y políticos como de los derechos económicos sociales y culturales.

$110 \quad$ Walter Kalin, Displacement Caused by the Effects of Climate Change: Who will be affected

and what are the gaps in the normative frameworks for their protection? Background Paper submitted by the Representative of the Secretan' General on the Human Rights of Internally Displaced Persons, Oslo, Norway, October 2008.

111 Global Protection Cluster Working Group, Handbook for the Protection of Internally Displaced

Persons, Marzo de 2010, pág. 12. 
Los desastres y el desplazamiento "pueden afectar a los individuos de diferentes maneras dependiendo de factores como la edad, el género y otras características étnicas, sociales y religiosas" ${ }^{\prime 112}$. Ciertos grupos de personas que pueden ser afectadas por un desastre como las mujeres, las niñas, los adulos mayores o las personas con discapacidad se encuentra en ciertas circunstancias marginados del proceso de toma de decisiones, y como resultado sus necesidades específicas de protección desatendidas.

La protección de las personas en contexto de desastres requiere necesariamente del empleo de un enfoque de edad, género y diversidad para la identificación y respuesta frente a los riesgos de protección de sus derechos humanos. Al respecto, el "fracaso en reconocer los riesgos particulares que enfrentan los diversos miembros de una comunidad no solo resulta en el fracaso de las medidas dirigidas a esas preocupaciones, sino que también puede conducir a acciones que inadvertidamente aumentan esos riesgos y refuerzan la discriminación y la exclusión"113.

\subsection{Riesgos de Protección}

La experiencia recogida en el terreno de la labor humanitaria en el marco de los grandes desastres naturales de los últimos años ha permitido recabar información, lecciones y algunos patrones acerca de la importancia de adoptar acciones para la protección de los derechos humanos de la persona afectada. Las Directrices Operacionales del Comité Permanente entre Organismos (IASC) en materia de protección en contexto de desastres naturales, tanto en su versión del año 2006 como en la versión definitiva publicada a comienzos del 2011, constituyen un aporte fundamental a la promoción de un enfoque basado en los derechos humanos en situaciones de desastres naturales.

Este documento del IASC se constituye en una verdadera guía de trabajo para la protección de los derechos humanos en el contexto de los desastres naturales. Uno de sus principales aportes radica en la identificación de ciertos derechos cuyo goce y ejercicio se ve particularmente afectado en el contexto de los desastres. Los grupos de derechos identificados son:

A- derechos relacionados con la vida; la seguridad e integridad física, y la protección de los lazos familiares;

B- derechos relacionados con la provisión de alimentos; servicios de salud, alojamiento; y educación;

C- derechos relacionados con la vivienda, la tierra, la propiedad y los medios de subsistencia;

D- derechos relacionados con la documentación, la libre circulación en el contexto de soluciones duraderas para los desplazados internos, restablecimiento de los lazos familiares, expresión y opinión, y elecciones.

A priori, es posible señalar que las situaciones y derechos correspondientes a los dos primeros grupos cobran particular relevancia en la fase de respuesta al de-

$112 \quad$ Op. cit. Global

Displaced Persons, Marzo de 2010,pág. 12

113 Op. cit. Global

Displaced Persons, pág. 13.
Protection Cluster Working Group. Handbook for the Protection of Internally

Protection Cluster Working Group, Handbook for the Protection of Internally 
sastre, mientras que los derechos contemplados en los dos grupos restantes habrán de cobrar mayor relevancia en la fase de reconstrucción o recuperación.

La protección de los derechos enumerados en el primer grupo, vida, seguridad, integridad física, cobra especial relevancia en contextos dominados por la existencia de amenazas directas a la seguridad de las personas. Algunas de estas amenazas provienen específicamente de los eventos que ocasionan el desastre, mientras que otras surgirán de las condiciones de supervivencia que las personas deberán sobrellevar como consecuencia del desastre.

En este sentido, las acciones de protección abarcan un abanico amplio de actividades que pueden ir desde las medidas de evacuación de población desde áreas riesgosas, al establecimiento de campamentos o centros de albergue de emergencia y la consiguiente necesidad de implementar medidas para la preservación de la seguridad en dichos ámbitos, ya que la precariedad que generalmente impera en los campamentos y la competencia por bienes esenciales muchas veces escasos como agua, vestido y alimento puede conducir a disputas que pongan en riesgo la seguridad de las personas.

En relación a las medidas de evacuación y establecimiento de campamentos, se debe hacer una mención especial a la situación de las personas con discapacidad. Por lo general, éstas "tienen más probabilidades de quedarse atrás o ser totalmente abandonadas durante una evacuación en caso de desastre y conflicto debido a la falta de preparación y planificación, así como a la inaccesibilidad de las instalaciones y los servicios y de los sistemas de transporte" ${ }^{114}$. La atención de las necesidades de las personas con discapacidad en contextos de desastre ha sido recogida expresamente en la Convención sobre los derechos de las personas con discapacidad de las Naciones Unidas aprobada en diciembre de 2006. En el artículo 11 de la Convención, relativo a situaciones de riesgo y emergencias humanitarias, se presta especial atención a la obligación de los Estados Partes de adoptar "todas las medidas necesarias para garantizar la seguridad y la protección de las personas con discapacidad en situaciones de riesgo, incluidas situaciones de conflicto armado, emergencias humanitarias y desastres naturales".

Bajo este primer grupo de derechos incluido en las Directrices, también se aborda la protección de las mujeres y niñas contra la violencia sexual y de género. La experiencia en el trabajo con desplazadas internas, incluidos los casos de mujeres desplazadas por los desastres naturales, demuestra que "allí donde la ley y el orden se quiebran, donde las redes de seguridad que proveen la familia y la comunidad desaparecen, las mujeres se tornan más vulnerables a sufrir la violación de sus derechos, incluida la violencia física y sexual"115. Los campamentos o centros de evacuación que albergan a los desplazados internos representa "un microcosmos de las relaciones disfuncionales de género"116 en el marco del cual el estatus subordinado de la mujer la coloca frente a mayor riesgo de sufrir situaciones de violencia y abuso. La violencia

114

ENABLE, Discapacidad, desastres naturales y situaciones de emergencia: La necesidad de incluir a las personas con discapacidad, disponible en: http://www.un.org/spanish/disabilities/default. asp?id $=1552$.

115 Declaraciones de la vocera de la Coalition for Assisting Tsunami Affected Women (CATAW) al sitio web AsiaNews.it, 26 de enero de 2005.

116 Rees, Susan, Pittaw'ay, Eileen y Bartolomei, Linda, llares of Violence: Women in Post-Tsunami Sri Lanka, The Australasian Journal of Disaster and Trauma Studies, Volume : 2005-2, 2005, disponible en: http://www.massey.ac.nz/-trauma/issues/2005-2/rees.htm. 
sexual y de género que afecta a las mujeres desplazadas representa una clara muestra de la exacerbación de las situaciones de discriminación y vulnerabilidad preexistentes a las que referimos anteriormente.

Tras el tsunami que afectó gran parte del sudeste asiático a finales de 2004, la violencia sexual se convirtió en uno de las principales amenazas a la vida y seguridad de las mujeres y niñas que habían sobrevivido. Incidentes de violación, violación engrupo, acoso y abuso físico de mujeres y niñas fueron reportados durante la fase de respuesta inmediata así como durante la residencia de éstas en campamentos y centros de evacuación"7. La violencia sexual también se trasformó en uno de los principales desafíos de protección en el Haití post terremoto. La violencia sexual en contra de mujeres y la violencia y el abuso en contra de niñas y niños representa una de las principales amenazas a los derechos humanos en los campamentos que albergan a los desplazados internos" ${ }^{8}$.

Generar conciencia entre las mujeres acerca de su derecho a una vida libre de violencia y los procedimientos de denuncia, trabajar en la sensibilización de hombres y niños, trabajar con las autoridades y las mujeres en fortalecer la iluminación y seguridad en áreas sensibles de los campamentos o centros del albergue colectivo como los baños, duchas o áreas linderas inseguras, constituyen tan solo algunas de las acciones que se deben emprender para la protección de las mujeres y niñas frente al riesgo de la violencia sexual y basada en género" ${ }^{9}$.

El alimento, el agua y el acceso a servicios de salud resultan fundamentales para asegurar la plena vigencia de los derechos humanos. Los desastres naturales impactan en el normal funcionamiento de la comunidad afectando el acceso de las personas a bienes y servicios esenciales que aseguran el ejercicio de estos derechos. Los desastres suelen afectar la estructura y capacidad de respuesta de los servicios de salud, también pueden afectar las redes de distribución de agua potable y saneamiento. El acceso al alimento también se convierte en un problema y en numerosos casos las personas más vulnerables pasan a depender de la distribución de alimento por parte de agencias humanitarias para poder sobrevivir. Son precisamente estas situaciones las que se abordan en el segundo grupo de derechos incluido en las Directrices de Protección.

El desafío radica en asegurar el acceso de todas las personas afectadas por los desastres a estos bienes y servicios humanitarios sin discriminación y en condiciones que aseguren su dignidad y la protección contra la explotación. Particular atención entre otros: Vella, Danielle, Violence against women continúes after the Tsunami. Asia News, 26 de enero de 2005. disponible en: http://www.asianews.it/index.php?l=en\&art=2427; OXFAM Internacional. Oxfam Briefing Note: The tsunami $s$ impact on women, marzo de 2005, disponible en: http://www.oxfam. org.uk/resources/policy/conflict_disasters/downloads/bn tsunami_women.pdf.

118 Cfr. Misión de Estabilización de las Naciones Unidas en Haití (MINUSTAH). IDPcamp: Joint Security Assessment report. MINUSTAH Human Rights Section. 30 de marzo de 2010.

$119 \quad$ El riesgo de violencia sexual y basada en género al que se encuentran expuestas las mujeres y niñas desplazadas por los desastres naturales no se diferencia demasiado de los riesgos que afrontan las mujeres y niñas refugiadas o desplazadas internamente por efecto de los conflictos. Para más información sobre la protección de las mujeres y niñas se recomienda acudir a: ACNUR, Violencia sexual y por motivos de género en contra de personas refugiadas, retornadas $v$ desplazadas internas - Guía para la preven-

ción y respuesta,2003, disponible en: http://www.acnur.org/t3/fileadmin/scripts/doc.php?file=biblioteca/ pdf/3667; y ACNUR. Manual para la Protección de Mujeres y Niñas, 2008, disponible en: http://www. acnur.org/t3/fileadmin/scripts/doc.php?file=biblioteca/pdf/7137. 
requiere la situación de grupos vulnerables o de personas con necesidades específicas como las personas con discapacidad, las personas viviendo con VIH/SIDA y los adultos mayores. La disponibilidad, accesibilidad, aceptabilidad y adaptabilidad de los bienes y servicios humanitarios resultar ser condiciones esenciales para asegurar a las personas afectadas por un desastre natural su derecho a una alimentación adecuada, el derecho al agua y saneamiento, así como el derecho a alojamiento y el derecho a la salud.

El caso de las personas con discapacidad se toma especialmente relevante al momento de planificar los sistemas y mecanismos de distribución de la asistencia humanitaria. Uno de los principales y más comunes errores en esta área consiste en pensar que todas las personas afectadas por el desastre pueden desplazarse por sus propios medios y aguardar una fila para acceder a la distribución de agua, alimento o vestimenta. Los discapacitados muchas veces no pueden hacerlo.

El caso de las personas viviendo con VIH/SIDA resulta de particular atención. Los desastres naturales pueden interrumpir el tratamiento médico que las personas viviendo con H1V requieren para el cuidado de su salud. Asimismo, los desastres naturales pueden afectar la calidad del agua y del alimento generando aún mayores riesgos. Asegurar el pronto restablecimiento de los tratamientos retrovirales y el acceso a agua y alimentación adecuada se torna en una acción imprescindible para asegurar la protección de sus derechos fundamentales. Una situación en alguna medida similar se plantea respecto de los adultos mayores en tanto pueden requerir de cuidados médicos y una alimentación diferenciada para poder sobrevivir.

Como claramente lo sostuvo el Secretario General de la Federación Internacional de Sociedades de la Cruz Roja y la Medialuna Roja (1FRC, por sus siglas en inglés), "los desastres naturales localizan a los pobres y se aseguran que estos continúen siendo pobres"12". Un dato estadístico respalda esta afirmación. De acuerdo al Programa de las Naciones Unidas para el Desarrollo (PNUD), "los países que registran un alto desarrollo humano albergan al 15 por ciento de la población expuesta, pero sólo sufren un 1,8 por ciento de las muertes originadas por los desastres ${ }^{\prime 121}$, mientras que los 50 países menos desarrollados del planeta a pesar de albergar solo al 11 por ciento de la población expuesta, aportan alrededor del $53 \%$ de las víctimas fatales por desastres naturales.

Resulta preciso señalar que "la vulnerabilidad humana a las amenazas naturales y la pobreza por bajos ingresos se encuentran íntimamente relacionadas". En muchos casos, "reducir el riesgo de desastre está a menudo supeditado a paliar la pobreza y viceversa"122. Las personas pobres no solo se ven mayormente expuestas a los efectos adversos de los desastres naturales, sino que además ven generalmente agravada su situación de pobreza como consecuencia de éstos. Los pobres tienen mucho para perder en un contexto de desastres.

El tercer grupo de derechos reconocido en las Directrices alude precisamente a la protección de los derechos relacionados a la vivienda, la tierra, la propiedad, los

120 Cherpitel, Didier, Breaking the cycle. Our Planet Vol. 11 No. 3: Disasters, 2002, disponible en: http://www.ourplanet.eom/imgversn/l 13/cherp.html .

121 Programa de las Naciones Unidas para el Desarrollo (PNUD), Un Informe Mundial La Reducción de Riesgos de Desastres: Un Desafio para el Desarrollo, PNUD, Nueva York, EEUU, 2004, pág. 3.

122 Op. cit. PNUD, Un Informe Mundial. La Reducción de Riesgos de Desastres: Un Desafio para el Desarrollo, pág. 16. 
medios de subsistencia y la educación secundaria y superior. La protección de estos derechos resulta fundamental en las fases de rehabilitación y reconstrucción a fin de mitigar el efecto de los desastres naturales en las condiciones de subsistencia de las personas y grupos que ya de por sí eran vulnerables antes del evento y reducir su riesgo de exposición a nuevos desastres en el futuro.

Proteger la propiedad y los medios de subsistencia de los que menos tienen resulta fundamental para prevenir la marginalidad, exclusión y la violación de derechos humanos fundamentales. La protección de tales derechos requiere necesariamente la adopción de medidas para asegurar que las personas, pueblos o comunidades afectadas y/o desplazadas por un desastre natural puedan recuperar las posesiones y propiedades que debieron dejar atrás y la protección contra los saqueos, la destrucción, la apropiación, ocupación o uso arbitrario de las mismas, tanto por parte de terceros como de agentes del Estado. Los medios de subsistencias "permiten a las personas ganarse el sustento [y] abarcan las capacidades, los bienes, los ingresos y las actividades de las personas necesarios para asegurar que se cubren sus necesidades vitales" ${ }^{123}$. Se vinculan de modo directo con el derecho a trabajar y a una vida digna.

Los planes de rehabilitación frente a una situación de desastre deben necesariamente incorporar acciones dirigidas a proteger el derecho a la propiedad de los afectados incluyendo "el acceso de los propietarios a recursos eficaces para presentar denuncias contra personas que ocupan o usan ilegalmente su propiedad" y "el establecimiento de procedimientos simplificados para la restitución de títulos de tierras o documentos de propiedad"124.

Un caso de particular representa el de los territorios o tierras comunitarias campesinos e indígenas que suelen ubicarse en zonas altamente expuestas a los efectos adversos de los desastres. Un estudio de FLACSO afirma que "los desastres naturales y sus efectos, agudizarán más la pobreza de los Pueblos Indígenas" que por lo general se "ven forzados a ocupar los lugares más riesgosos para vivir y trabajar" 125. La protección de la integridad de las tierras comunitarias campesinas e indígenas no sólo se vincula a la protección del derecho a la propiedad individual de cada uno de sus miembros, sino que tiene un impacto fundamental en al protección de la identidad cultural, comunitaria y hasta en la misma subsistencia de los pueblos indígenas. En estos casos, la tierra juega un rol fundamental para "mantener y reforzar sus instituciones, culturas y tradiciones y promover su desarrollo de acuerdo con sus aspiraciones y necesidades"126. Promover la recuperación de las tierras y la restitución de los títulos que pudieron haberse destruido se toma una acción fundamental. En los casos que las tierras se hayan tomado inhabitables, se deberá evaluar al reubicación de la comunidad de su conjunto con el objeto de preservar la vida e identidad comunitaria.

En materia de subsistencia, la recomendación pasa por asegurar "el acceso a oportunidades de subsistencia y empleo, así como a los proyectos para restaurar las recuperacion/que-son-los-medios-de-subsistencia/.

124 Op. cit. IASC, Directrices Operacionales sobre la protección de las personas en situaciones de desastres naturales. Proyecto de Brookings - Bem sobre Desplazamiento Interno, Mayo de 2011, págs. 41 y 42 .

125

Lux de Cotí, Otilia, Gobernabilidad v Pueblos Indígenas, FLACSO, San José, Costa Rica,

2010, pág. 10.

126

Naciones Unidas Asamblea General, Declaración de las Naciones Unidas sobre los derechos

de los pueblos indígenas, adoptada por la Asamblea General de Naciones Unidas el 2 de octubre de 2006. 2007, $\mathrm{A} / \mathrm{RES} / 61 / 295$. 
actividades económicas, las oportunidades de empleo y los medios de subsistencia que se ven interrumpidos por el desastre natural, sin discriminación alguna, lo antes posible y del modo más exhaustivo posible" ${ }^{127}$. Los desastres naturales barren en muchos casos con los medios de vida de las personas afectadas. Así como los tsunamis han demostrado su capacidad devastadora para arruinar al sector pesquero, las inundaciones y sequías pueden afectar a los productores rurales, arruinando conjuntamente a las economías locales que subsisten en tomo de estas actividades. La no inclusión de programas y proyectos para rehabilitar las oportunidades de subsistencia condena a las personas afectadas a la pobreza y a sobrevivir de las donaciones de las agencias humanitarias.

La documentación personal no debiera resultar nunca un obstáculo para el acceso a derechos fundamentales como la alimentación, la educación o la salud. Sin embargo, en la práctica, aparece muchas veces como un requisito para el acceso a políticas y programas públicos sociales. También la documentación se toma necesaria para poder acceder a un empleo formal, abrir y operar una cuenta bancaria, celebrar un contrato o incluso presentar un reclamo judicial. En el contexto de los desastres, las personas pueden perder o ver destruida toda su documentación y certificados personales (nacimiento, matrimonio, etc). En este sentido, "la adopción rápida de procedimientos administrativos simplificados para la re-emisión de la documentación personal"128 se toma en una medida esencial para la salvaguarda de derechos.

La libertad de circulación constituye otro importante derecho cuyo ejercicio se encuentra directamente asociado a la búsqueda de una solución duradera para las personas desplazadas por efecto de un desastre natural. Superada la emergencia, las personas afectadas por el desastre "deberán tener el derecho a elegir libremente si quieren regresar a sus hogares y lugares de origen, integrarse localmente en el área a la que han sido desplazados, o reasentarse en cualquier otro lugar del país" ${ }^{129}$. En cualquiera de estos escenarios, deberán adoptarse las medidas para asegurar que la solución en cada caso se de en un marco que resguarde la seguridad y dignidad de las personas.

La experiencia recogida en el terreno arroja un panorama claro y a la vez crudo acerca del impacto devastador que los desastres naturales pueden llegar a tener en el goce y ejercicio de los derechos humanos de las personas afectadas. La incorporación de un enfoque basado en derechos y de iniciativas de protección de los derechos humanos en los planes de prevención y respuesta a desastres constituye un desafío humanitario fundamental y a la vez una obligación a la cual los Estados deben atender como parte de su obligación general de respetar y garantizar los derechos humanos.

\section{EL IMPACTO PEI, CAMBIO CLIMÁTICO EN LA LOS}

Un terremoto, una inundación o un tsunami pueden en un plazo de tiempo verdaderamente corto arrojar a miles de personas a la calle, forzarlos a vivir en paupérrimo

\footnotetext{
127 Op. cit. IASC, Directrices Operacionales sobre la prolección de las personas en siiuuciunes de desastres naturales, Proyecto de Brookings - Bem sobre Desplazamiento Interno, Mayo de 2011, pág. 44.

128 Op. cit. IASC, Directrices Operacionales sobre la protección de jas personas en situaciones de desastres naturales. Proyecto de Brookings - Bem sobre Desplazamiento Interno, Mayo de 2011. pág. 47.

129 Op. cit. IASC, Directrices Operacionales sobre la protección de las personas en situaciones de desastres naturales, Proyecto de Brookings - Bem sobre Desplazamiento Interno, Mayo de 20 U, pág. 49.
} 
condiciones de asistencia y seguridad en campamentos, arruinar por completo sus propiedades y medios de subsistencia, y afectar gravemente la infraestructura de educación y salud de la comunidad en la que viven. Como se analizo a lo largo de la primera parte de este trabajo, el vínculo entre los desastres naturales y la amenaza a los derechos humanos es clara y evidente. Sin embargo, esa vinculación no resulta tan evidente frente a los efectos adversos del cambio climático de proceso lento.

En 2008, El Consejo de Derechos Humanos de las Naciones Unidas reconoció "el cambio climático crea una amenaza inmediata y de gran alcance para la población y las comunidades de todo el mundo y tiene repercusiones sobre el pleno disfrute de los derechos humanos"130. Un Informe hecho público en 2009 por la Oficina del Alto Comisionado de las Naciones Unidas para los Derechos Humanos $(\mathrm{OACNUDH})$ a pedido del Consejo aborda el estrecho vínculo que existe entre el medio ambiente y el goce efectivo de una serie de derechos humanos.

El derecho a la vida y la seguridad de las personas se verá severamente afectado por los desastres naturales que como consecuencia del cambio climático habrán de aumentar en frecuencia e intensidad, existen derechos amenazados cuya vigencia se ve amenazada por los efectos del cambio climático de proceso lento. Como se mencionó en la introducción a este trabajo, otros derechos humanos como el derecho a una alimentación adecuada, el derecho al agua, el derecho a la salud, el derecho a una vivienda adecuada y el derecho a la libre determinación se encuentran entre los derechos humanos que se verían severamente comprometidos por las consecuencias del cambio climático.

El aumento de la temperatura media mundial incidirá de manera determinante en la capacidad de producción de alimentos y en ciertas partes del mundo generará situaciones de riesgo e inseguridad alimentaria. Un ejemplo del impacto del cambio climático en el derecho a una alimentación adecuada se registra actualmente en la zona del Cuerno de África donde 4 millones de personas afrontan un situación de hambruna como consecuencia de una de las perores sequías en la historia de Somalia ${ }^{131}$.

El Relator Especial sobre el derecho a la alimentación ha señalado que "el cambio en la temperatura media está amenazando la capacidad de regiones enteras, particularmente las que viven de la agricultura de secano, para mantener los niveles actuales de producción agrícola". Asimismo, la menor disponibilidad de agua para riego y la salinización del agua en las zonas costeras hacen prever que "para el año 2080, otros 600 millones de personas podrían correr peligro de padecer hambre, como consecuencia directa del cambio climático"132.

El derecho al agua, entendido como el derecho de toda persona a disponer de agua suficiente y salubre para usos personales y domésticos como el consumo, la cocina y la higiene personal y doméstica ${ }^{133}$, se verá afectado por la pérdida de los

\footnotetext{
130 Naciones Unidas Consejo de Derechos Humanos, Resolución 7/23 Derechos humanos y cam-

bio climático, 41 " sesión, 28 de marzo de 2008

131 Oficina para la Coordinación de Asuntos Humanitarios (OCHA), Somalia $\bullet$ Famine \& Droughl. Silualion Repon No. 20, OCHA. 1 de noviembre de 2011.

$132 \quad$ Naciones Unidas Consejo de Derechos Humanos, Informe del Relator Especia! sobre el dere cho a la alimentación. Sr. Olivier De Schutter, A/HRC/16/49, 20 de diciembre de 2010, páarfo 9.

$133 \quad$ Naciones Unidas Comité de de Derechos Económicos, Sociales y Culturales, Observación General No. 15 (2002). El derecho al agua (artículos II y 12 del Pacto Internacional de Derechos Económicos, Sociales y Culturales) E/C. 12/2002/11, 20 de enero de 2003.
} 
glaciares y la reducción de capa de nieve que afectarán la disponibilidad de agua de deshielo de las cadenas montañosas. Se estima que el cambio climático agravará en las próximas décadas el problema del acceso al agua potable que hoy en día afecta a alrededor de 100 millones de personas en todo el mundo.

El derecho Internacional reconoce el disfrute del más alto nivel posible de salud física y mental como un derecho humano fundamental ${ }^{134}$. Las predicciones sobre los efectos del cambio climático alertan sobre el impacto en la salud de millones de personas a través del aumento de la desnutrición y la propagación d enfermedades diarreicas, cardiorrespiratorias e infecciosas. Asimismo, el cambio climático agravará la situación de los sistemas de salud en todo el mundo condicionando el acceso a los servicios de salud de cientos de miles de personas. ${ }^{135}$

El derecho a la libre determinación se encuentra reconocido en el derecho internacional como la potestad de los pueblos de establecer libremente su condición política y proveer a su desarrollo económico, social y cultural ${ }^{136}$.

Una de las dimensiones de la amenaza del cambio climático a la libre determinación de los pueblos está dada por la delicada situación que enfrentan los estados insulares que, como Las Madivas, Tuvalu, Kiribati o las Islas Marshall, corren el riesgo de tomarse completamente inhabitables o directamente desaparecer bajo el agua ante el aumento general del nivel del mar como consecuencia del calentamiento global y el consecuente derretimiento de los cascos polares. La simple existencia de estos pequeños Estados insulares se ve amenazada por efecto del cambio climático. Distintas previsiones afirman que "en el futuro la población total de los Estados a nivel del mar como las Maldivas, Tuvalu, Kiribati y las Islas Marshall podría estar obligada a dejar su propio país como resultado del cambio climático" y "la existencia como tal de sus Estados podría estar amenazada"137.

Otra dimensión del impacto del cambio climático en la libre determinación está dada por la vinculación entre el cambio climático y los conflictos armados. El cambio climático juega un papel importante en profundizar algunos conflictos existentes y generar situaciones propicias para la ocurrencia de nuevos conflictos. En varias partes del mundo, la disputa por recursos cada vez más escasos como el agua potable o la tierra cultivable se combinan con factores étnicos y sociales para potenciar el riesgo de conflicto o agravar las tensiones ya existentes. Un reporte de la organización Internacional Alert del año 2007 señala que existen 46 países en el mundo -que albergan más de 2,7 mil millones de personas- en los cuales el efecto del cambio climático en interacción con problemas económicos, sociales y políticos puede conducir a un alto riesgo de conflictos violentos ${ }^{138}$. Pacto Internacional de Derechos, Económicos, Sociales y Culturales y 10 del Protocolo Adicional a la Convención Americana sobre Derechos Humanos en materia de Derechos Económicos, Sociales y Culturales (Protocolo de San salvador). Referencias al derecho a la salud también se hallan en otros instrumentos internacionales de derechos humanos.

135 Op. Cit. Naciones Unidas Consejo de Derechos Humanos,

Informe de la Oficina deI Alto Co

misionado de las Naciones Unidas para los Derechos Humanos (OACNUDHl sobre la relación entre el cambio climático y los derechos humanos, párrafos 32 y 33 .

136 Internacional de Derechos Civiles y Políticos; y la Declaración sobre el Derecho al Desarrollo.

137 ACNUR. Cambio climático y apatrida: una visión general,

wwvl.unhcr.org/refworld/docid/4adec0eb2.html.

138 Smith, Dan \& Vivekananda, Janani, A Climate of Conflict.

peace and war. Internacional Alert, Noviembre de 2007
15 May 2009. disponible en: http://

The links behveen climate change, 
Un informe del Secretario General de las Naciones Unidas sobre el cambio climático y sus posibles repercusiones para la seguridad advierte que "en los lugares donde se prevé que el cambio climático constituirá una grave amenaza para el bienestar humano, en particular los lugares en que la gente es especialmente vulnerable debido al bajo nivel de desarrollo humano y a la fragilidad de las instituciones de gobierno, las repercusiones para la seguridad tienden a ser más pronunciadas, incluida la posibilidad de que se produzcan tensiones sociales y políticas y conflictos armados" 139 .

La exposición a los efectos adversos del cambio climático también debe ser analizada desde un enfoque sensible al género, la edad y la diversidad. El Informe de la OACNUDH afirma expresamente que "Ios efectos del cambio climático se dejarán sentir con más fuerza en los segmentos de la población que ya se encuentran en situación vulnerable debido a factores como la pobreza, el género, ladead y la condición de minoría y la discapacidad"140. Las mujeres, los niños, los pueblos indígenas se encuentran entre los grupos de mayor exposición a las amenazas a los derechos humanos derivados del cambio climático.

El impacto del cambio climático en el goce y ejercicio de los derechos humanos ya se siente con fuerza en diversas partes del mundo y, de acuerdo a los pronósticos y predicciones científicas disponibles, se agravará en el transcurso de las próximas décadas haciéndose sentir con mayor fuerza en al realidad de los grupos ya de por si vulnerables. Asimilar esta realidad e incorporar acciones concretas en los planes de adaptación al cambio climático para mitigar su impacto en los derechos humanos constituye un imperativo ético y de derecho para los Estados y la Comunidad Internacional en el marco de la vigencia del derecho internacional de los derechos humanos.

\section{BESASTRES-NATURAHES. CAMBЮ-CHMATHO-}

\section{DESPLAZAMIENTO}

El estudio del impacto de los desastres naturales y el cambio climático en el desplazamiento forzado de personas y los desafíos que este fenómeno plantea en términos humanitarios y de derechos humanos requiere de un abordaje profundo y cuidadoso acerca de sus implicancias legales, sociales, culturales y políticas que no serán objeto de este trabajo. Sin embargo, no se puede dejar de mencionar que el desplazamiento forzado se constituye en uno de las principales y más complejas consecuencias humanitarias de los desastres naturales y el cambio climático.

Ya en el año 1990, el primer reporte de evaluación del Panel Intergubernamenta sobre el Cambio Climático (IPCC, por sus siglas en inglés) había alertado que una de las principales consecuencias del cambio climático se daría en el ámbito de la migración humana. En el informe se estimaba que para el año 2050, 150 millones de personas se habrían desplazado como consecuencia de fenómenos y desastres naturales asociados al cambio climático ${ }^{141}$.

misionado de las Naciones Unidas para los Derechos Humanos (OACNUDH) sobre la relación entre el cambio climático y los derechos humanos, párrafo 42.

141 Change, IPCC) fue establecido en el año 1988 por la Organización Meteorológica Mundial (World Meteo - rological Organization, WMO) y el Programa Ambiental de las Naciones Unidas (United Nations Environ- 
El desplazamiento como consecuencia del cambio climático y los desastres naturales aparece como "parte normal de la adaptación al cambio, proveyendo un medio para escapar al peligro e incrementar la resiliencia" ${ }^{142}$. El desplazamiento se transforma así en una estrategia válida de supervivencia frente a la amenaza que el cambio climático y los desastres naturales imponen a la vida, seguridad y otros derechos fundamentales de las personas. El carácter forzado del desplazamiento lleva implícita la falta de opciones y de libertad al momento de tomar la decisión, "la migración forzada conlleva falta de consentimiento, la negación o una disminución de la libertad de agencia"143.

Un informe presentado en 2011 por el Centro para el Monitoreo del Desplazamiento Interno (IDMC, por sus siglas en inglés) en el marco de la Conferencia Nansen sobre el Cambio Climático y el Desplazamiento en el Siglo XXI alertó sobre la presencia de más de 43 millones de personas desplazadas en el mundo como consecuencia de los efectos adversos del cambio climático y los desastres naturales ${ }^{144}$. El informe estima en 42.3 millones el número de personas desplazadas como consecuencia de desastres en 2010, 38.3 millones de desplazados como consecuencia de desastres asociados a factores climáticos mientras que tan solo 4 millones fueron desplazados como consecuencias de desastres asociados a terremotos y otros eventos de origen geológico. El mayor número de los nuevos casos de desplazamiento registrados en 2010 se dieron en Asia: 77\%, mientras que América se colocó en un segundo lugar con alrededor del $19 \%$ de los casos, alrededor de 8 millones de desplazados.

La mayor parte de las personas desplazadas como consecuencia de los efectos del cambio climático y los desastres naturales habrá de permanecer dentro de las fronteras del territorio de sus propios Estados. El informe del IDMC no distingue en sus estimaciones entre personas desplazadas internamente dentro de sus propios Estados y personas que se hubieran desplazado a través de una frontera internacionalmente reconocida. Sin embargo, el propio informe sostiene que "la evidencia disponible sugiere que a nivel global la gran mayoría de desplazados por desastres naturales permanecen como desplazados internos"145.

Las personas que como consecuencia del cambio climático y los desastres naturales se desplazan dentro del territorio de su propio Estado caen bajo el ámbito de la definición de "desplazados internos". De conformidad con los Principios Rectores de los Desplazamientos Internos, se entiende por desplazados internos a:

"las personas o grupos de personas que se han visto forzadas u obligadas a escapar o huir de su hogar o de su lugar de residencia habitual, en particular

ment Programme, UNEP) con el objetivo de analizar la información científica, técnica y socioeconómica relevante para la comprensión de los elementos científicos relativos al cambio climático de origen antropogénico así como sus posibles repercusiones, riesgos y sus posibilidades de atenuación y de adaptación 142 McAdam, Jane, Swimming againsl the Tide: Why a Climate Change Displacement Treaty is No the Answer, International Journal of Refugee Law, Vol. 23 No. I, enero de 2011, pág. I.

143 Corlett. David, Stormv Weather. The Challenge of Climate Change and Displacement, Univer sity of New South Wales, Sydney, Australia, 2008, pág. 43.

$144 \quad$ Intemal Displacement Monitoring Center (IDMC), Displacement dne to natural hazard-in duced disasters: Global estimates for 2009 and 2010, pág. 4, Junio de 2011, disponible en: http://www. intemal-displacement.org/8025708F004BE3B 8A/\$file/IDMC natural-disasters_2009-2010.pdf.

145 Op. cit. IDMC, Displacement due to natura! hazard-induced disasters: Global estimates for 2009 and 2010, op. cit., página 7. 
como resultado o para evitar los efectos de un conflicto armado, de situaciones de violencia generalizada, de violaciones de los derechos humanos o de catástrofes naturales o provocadas por el ser humano, y que no han cruzado una frontera estatal internacionalmente reconocida".

A pesar de no ser una norma de carácter legalmente vinculante para los Estados como lo sería un tratado, la validez legal de los Principios Rectores de los Desplazamientos Internos ha sido ampliamente reconocida en resoluciones de la Comisión de Derechos Humanos (actual Consejo de Derechos Humanos), el Consejo Económico y Social de las Naciones Unidas (ECOSOC), y principalmente por la práctica y legislación de los Estados y agencias humanitarias que los han aplicado a la hora de legislar y adoptar medidas para prevenir y responder al desplazamiento interno.

En el ámbito interamericano, la importancia central de los Principios Rectores de los Desplazamientos Internos para la protección y atención de las necesidades de los desplazados ha sido reconocida por diversas Resoluciones de la Asamblea General de la Organización de Estados Americanos (OEA) que incluso ha solicitado expresamente a los Estados que "en la atención a los desplazados internos en situaciones de desastres naturales y provocados por el ser humano, protejan sus derechos humanos mediante un enfoque integral para el socorro en casos de desastre y la reconstrucción" ${ }^{146}$.

Los Principios Rectores de los Desplazamientos Internos se basan en normas internacionales de carácter vinculante para los Estados, recogen principios y estándares del derecho internacional de los derechos humanos, el derecho internacional humanitario $y$, por analogía, del derecho internacional de refugiados' ${ }^{47}$. Los principios identifican los derechos y obligaciones de protección en las distintas fases o etapas del desplazamiento: etapa previa al desplazamiento, durante el desplazamiento, y durante la etapa de retomo, reasentamiento o reintegración. Los Principios Rectores reafirman los derechos humanos de las personas internamente desplazadas y clarifican las obligaciones de los Estados respeto a la protección de esos derechos. También juega un rol importante en orientar la labor de las agencias internacionales, organizaciones no gubernamentales y otros actores no estatales comprometidos en la tarea de atender y asistir a los desplazados.

Los Principios Rectores no reconocen nuevos derechos humanos a las personas desplazadas. Los desplazados internos continúan gozando de los derechos reconocidos en los catálogos internacionales de derechos humanos, como el Pacto Internacional de Derechos Civiles y Políticos y el Pacto Internacional de Derechos Económicos, Sociales y Culturales. El principal aporte de los Principios Rectores a la protección de los desplazados internos radica en clarificar el alcance y contenido de las obligaciones de los Estados para asegurar que esos derechos sean debidamente respetados y garantizados a través de las distintas etapas que se dan en situaciones de desplazamiento interno. Los Principios Rectores "adoptan el enfoque distintivo de reformular y adaptar los estándares legales internacionales a las especiales necesidades de los desplazados internos"148.

146 AG/RES. 2417 (XXXVI1I-O/08).

147 Cohén, Roberta, Introduction io the Guiding Principies on Internal Displacement, International Conference on Kurdish Refugees and Intemally Displaced Kurds, 23 de septiembre de 2001 . disponible en: http://wYvw.brookings.edu/speeches/2001/0923humanrights cohen.aspx.

148 Cohén, Roberta y Bradley, Megan, Disasters and Displacement: Gaps in Protection, Journal of

International Legal Studies, vol. 1, 2010, pág. 4. 
El principal desafío en materia de desplazamiento forzado como consecuencia del cambio climático y los desastres naturales se plantea respecto de las personas que se desplazan fuera del territorio de sus Estados de origen y que atraviesan en su afán por huir de los efectos adversos de estos fenómenos una frontera internacionalmente reconocida. La Oficina del ACNUR afirma que "tanto los desastres abruptos como los de proceso lento tienen el potencial para desplazar personas y comunidades, no sólo dentro del propio territorio de los Estados, sino también a través de fronteras internacionales" ${ }^{\prime 149}$.

A pesar de que el desplazamiento fue señalado desde hace varios años como una de las principales consecuencias humanitarias del cambio climático y los desastres que éste traería aparejados, la realidad es que todavía prima una total falta de claridad acerca del estatuto legal y la atención de las necesidades de protección de las personas que se desplazan fuera de su país escapando de las consecuencias de los desastres y la degradación del ambiente como consecuencia del cambio climático.

La situación de éstos "desplazados externos" plantea un importante desafío de protección. Estas personas suelen permanecer en los países de acogida en un limbo legal, sin ninguna claridad o certeza acerca de su estatuto legal y de derechos. Esta desprotección legal se traduce en la ausencia del derecho a residir en el país de acogida, en la exposición al riesgo de expulsión o deportación, riesgo de sufrir detención migratoria, indocumentación y serios obstáculos al ejercicio de derechos fundamentales como la salud, el trabajo y la educación. La desprotección legal de las personas forzadas a desplazarse fuera de su país por causa de los desastres también puede exponerlas al riesgo de explotación laboral y sexual, particularmente en el caso de las mujeres y niñas.

Las personas que se desplazan a través de fronteras internacionales escapando de los efectos de los desastres naturales y el cambio climático no suelen calificar como refugiados bajo la Convención de 1951 sobre el Estatuto de los Refugiados y su protocolo de 1967. Por definición, los refugiados huyen de una situación de persecución que comprende abusos o daños graves de los derechos humanos y que pueden ser resultado de la actuación de las autoridades de un país como de agentes no estatales que actúan con la aquiescencia o ante la incapacidad del Estado. Además de un agente de persecución, la definición legal del término refugiado exige que dicha persecución pueda necesariamente asociarse a uno de los cinco motivos enunciados en la Convención: Raza, religión, nacionalidad, pertenencia a un grupo social u opinión política ${ }^{150}$.

Si bien los desastres naturales y el cambio climático pueden conllevar la violación de derechos humanos, su caracterización como persecución bajo la definición de refugiado continúa siendo compleja. Un serio problema se plantea al momento de identificar el agente de persecución, así como a la hora de determinar el nexo casual

149 ACNUR, "Forced Displáceme»! in Ihe Conlexl of Climate Change: Challenges for Status Un-

der Internacional Law", documento presentado al Grupo de Trabajo especial sobre la cooperación a largo plazo en el Marco de la Convención Marco de las Naciones Unidas sobre el Cambio Climático, 19 de mayo de 2009.

150 Para una mejor comprensión de los elementos de la definición de refugiados e recomienda ver:

ACNUR, Manual de Procedimientos y Criterios para Determinar La Condición de Refugiado en virtud de ja Convención de 1951 y el Protocolo de 1967 sobre el Estatuto de los Refugiados, 1992. 
con alguno de los motivos estipulados en la definición de refugiado ${ }^{151}$. El empleo de términos como "refugiados climáticos" o "refugiados ambientales" ha sido severamente cuestionado y desaconsejado por los expertos y las agencias internacionales y humanitarias dedicadas a la protección de los refugiados y desplazados. Dichos términos carecen por completo de contenido legal y su uso "podría socavar el régimen jurídico internacional para la protección del refugiado y crear confusión respecto a la relación entre cambio climático, degradación ambiental y migración" 152.

A fin de atender a la situación legal de las personas desplazadas a través de las fronteras por los desastres naturales y el cambio climático se han desarrollado distintos abordajes que van desde proponer la adopción de una convención internacional que aborde el desplazamiento como consecuencia de los desastres naturales y el cambio climático, a posiciones menos ambiciosas y más pragmáticas que plantean buscar en las normas de derechos humanos, y en particular en la obligación de no devolución de los Estados, una respuesta a la situación de las personas que se desplazan a través de fronteras internacionales.

Algunos Estados han adoptado respuestas de carácter local a las necesidades de protección de las personas desplazadas por el cambio climático y los desastres a través de incluir en su legislación nacional estatutos o visas humanitarias que permiten responder de manera pragmática a la situación de los desplazados mediante el reconocimiento del derecho a una residencia legal, el acceso a documentación y la posibilidad de ejercer derechos esenciales como al educación, la salud y el trabajo. Se destacan los casos de Argentina, Brasil, los Estados Unidos y algunos países nórdicos como Suecia y Finlandia que han incorporado la posibilidad de otorgar visas o estatutos de protección humanitaria a personas que huyen de los desastres naturales o de la degradación del ambiente como consecuencia del cambio climático.

\section{$V$ CONCLUSIONES}

Los desastres naturales y el cambio climático han dejado de ser una mera amenaza potencial a los derechos humanos para convertirse en un verdadero y real desafío humanitario que compromete las estructuras, conceptos e incluso algunas de las concepciones legales actualmente vigentes. El impacto de estos fenómenos en el goce y ejercicio de los derechos humanos de millones de personas en todo el mundo es ostensible y afecta una amplia gama de derechos reconocidos en los instrumentos internacionales de derechos humanos que los Estados se han comprometido a respetar y garantizar.

La aplicación de un enfoque basado en derechos humanos frente a los desastres naturales y el cambio climático lleva implícita la revisión de las prácticas y mecanismos de respuesta que tradicionalmente los Estados y las agencias humanitarias han aplicado frente a los efectos adversos que conllevan estos fenómenos. La necesidad de recurrir a estándares internacionales de derechos humanos para la programación y la medición del impacto de las operaciones humanitarias, la necesidad de un enfoque comunitarios que refuerce el carácter de sujeto de derechos de las personas y la necesidad de aplicar un enfoque sensible al género, la edad y al diversidad han de imprimir importantes transformaciones en las actividades de preparación y respuesta a desastres y en las

151

Me Adam, Jane, El Desplazamiento provocado por el cambio climático y el Derecho Interna-

cional, Documento presentado ante el evento paralelo al Diálogo del Alto Comisionado sobre los Desafíos en materia de Protección, Ginebra, Suiza, 8 de diciembre de 2010.

152 ACNUR, Cambio climático, desastres naturales y desplazamiento humano: la perspectiva del ACNUR, 14 de agosto de 2009, disponible en: http://www.unhcr.org/refworld/docid/4ad7471b2.html. 
estrategias de mitigación.

Incorporar un enfoque de derechos humanos frente a los desastres y el cambio climático implica asimismo incorporar las políticas estatales en la materia bajo el paraguas de la obligación genérica del Estado de respetar y garantizar los derechos humanos. Este accionar puede dotar de mayor previsibilidad a la respuesta del Estado y también proporcionar mecanismos para compeler al Estado a adoptar medidas de protección, particularmente en aquellos casos que involucran a grupos especialmente vulnerables.

La referencia a los derechos humanos también resulta útil y necesaria para lidiar con el desafío que los desastres naturales y el cambio climático implican en materia de desplazamiento forzado de personas. La aplicación de los principios Rectores de los Desplazamientos Internos a la situación de las personas que como consecuencia de los desastres o del cambio climático se desplazan dentro del territorio de sus países de origen implica necesariamente reforzar un abordaje a la situación de estas personas desde una mirada de derechos humanos.

El derecho internacional de los derechos humanos, a través del Principio de No Devolución o Non Refoulement recogido en diversos instrumentos universales y regionales de derechos humanos, también realiza un importante aporte a la búsqueda de una respuesta de protección a las personas que huyendo de los efectos de un desastre o del cambio climático han huido de sus países de origen y han atravesado una frontera internacional. La obligación internacional de abstenerse de devolver a una persona a condiciones en las cuales puede ver gravemente lesionados sus derechos fundamentales ha animado a diversos Estados de nuestra región y de otras partes del mundo a abordar la situación de estas personas a través del otorgamiento de visas y/o estatutos humanitarios. Este principio de derechos humanos también se encuentra en el origen de diversas iniciativas que desde el multilateralismo buscan abordar la cuestión, ya sea a través de una convención específica o de la adopción de una serie de principios similares a los adoptados en relación al fenómeno de los desplazados internos ${ }^{151}$ que sirvan como guía a los Estados. 